
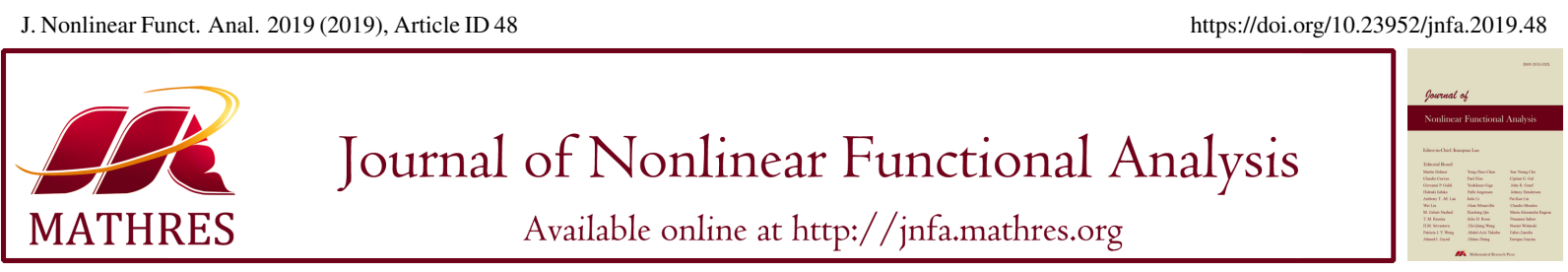

\title{
THE APPROXIMATION OF SOLUTIONS FOR GENERALIZED EQUILIBRIUM AND NONEXPANSIVE MAPPINGS
}

\author{
MEIJUAN SHANG \\ College of Science, Shijiazhuang University, Shijiazhuang 050035, China
}

\begin{abstract}
In this paper, we introduce and study a Bregan projection algorithm for treating generalized equilibrium and fixed point problems. Norm convergence of the projection algorithm is established in the framework of reflexive Banach spaces.
\end{abstract}

Keywords. Bregman projection; Convergence analysis; Equilibrium problem; Fixed point; Variational inequality. 2010 Mathematics Subject Classification. 65K15, 90C25.

\section{INTRODUCTION}

Let $E$ be a Banach space. Let $C$ be a nonempty convex and closed set in $E$ and let $E^{*}$ be the dual space of $E$. Let $M: C \rightarrow E^{*}$ be an operator and let $G: C \times C \rightarrow \mathbb{R}$ be a bifunction. Recall that the so called generalized equilibrium problem [1]: find $\tilde{x}$ such that

$$
\langle M \tilde{x}, y-\tilde{x}\rangle+G(\tilde{x}, y) \geq 0, \quad \forall y \in C .
$$

If $M=0$, then the generalized equilibrium problem is reduced to the classical equilibrium problem in the sense of Blum and Oettli [2]

$$
G(\tilde{x}, y) \geq 0, \quad \forall y \in C .
$$

If $G=0$, then the generalized equilibrium problem is reduced to the generalized variational inequality problem

$$
\langle M \tilde{x}, y-\tilde{x}\rangle \geq 0, \quad \forall y \in C .
$$

In this paper, the set of solutions of the generalized equilibrium problem is denoted by $G E P(M, G)$. The equilibrium problems provides us a a unified approach to investigate problems arising in many optimization problems; see [3, 4, 5, 6, 7, 8, 9] and the references.

E-mail address: meijuanshang@163.com.

Received June 28, 2019; Accepted December 16, 2019.

(C)2019 Journal of Nonlinear Functional Analysis 
For set $C, C B(C)$ is used to stand for the family of nonempty bounded closed subsets of $C$. Let $H(\cdot, \cdot)$ be the Hausdorff metric on $C B(C)$ defined by

$$
H(B, A)=\max \left\{\sup _{x \in A} d(x, B), \sup _{y \in B} d(y, A)\right\}, \quad \forall B, A \in C B(C),
$$

where $d(b, A)=\inf \{\|b-a\|: a \in A\}$ is the distance from point $b$ to subset $A$. Let $T$ be a set-valued mapping from $C$ to $C B(C)$. The set of fixed points of $T$ is denoted by $F(T):=\{p \in C: T(p)=p\}$. Let $f: E \rightarrow(-\infty,+\infty]$ be a proper, lower semi-continuous, and convex function. The domain of $f$ is presented by $\operatorname{dom} f$, i.e., $\operatorname{dom} f:=\{x \in E: f(x)<+\infty\}$. Let $\mathbb{R}$ and $\mathbb{N}$ be the sets of real numbers and positive integers, respectively. Let any $x \in \operatorname{int} \operatorname{dom} f$ and $y \in E$, the right-hand derivative of $f$ at $x$ in the direction of $y$ is defined by

$$
f^{\circ}(x, y)=\lim _{t \rightarrow 0^{+}} \frac{f(x+t y)-f(x)}{t} .
$$

Recall that the function $f$ is said to be Gâteaux differentiable if it is Gatteaux differentiable for any $x \in$ int $\operatorname{dom} f$; Gâteaux differentiable at $x$ if the limit $f^{\circ}(x, y)$ exists for any $y$; uniformly Fréchet differentiable on a subset $C$ of $E$ if the limit $f^{\circ}(x, y)$ is attained uniformly for $x \in C$ and $\|y\|=1$; Fréchet differentiable at $x$ if the limit $f^{\circ}(x, y)$ is attained uniformly in $\|y\|=1$. It is known if $f$ is Gâteaux differentiable at $x$, then $f^{\circ}(x, y)$ coincides with $\nabla f(x)$, the value of the gradient $\nabla f$ of $f$ at $x$. Let $x \in \operatorname{int} \operatorname{dom} f$, the subdifferential of $f$ at $x$ is the convex set defined by

$$
\partial f(x)=\left\{x^{*} \in E^{*}: f(x) \leq f(y)+\left\langle x^{*}, x-y\right\rangle, \quad \forall y \in E\right\} .
$$

The Fenchel conjugate of $f$ is the function $f^{*}: E^{*} \rightarrow(-\infty,+\infty]$ defined by

$$
f^{*}\left(x^{*}\right)=\sup \left\{\left\langle x^{*}, x\right\rangle-f(x): x \in E\right\}, \forall x^{*} \in E^{*} .
$$

In the framework of reflexive Banach spaces, we have the following facts: (i) $(\partial f)^{-1}=\partial f^{*}$ and $f$ is Legendre if and only if $f^{*}$ is Legendre; (ii) $f$ is essentially smooth if and only if $f^{*}$ is essentially strictly convex; (iii) If $f$ is Legendre, then $\nabla f$ is bijection satisfying $\nabla f=\left(\nabla f^{*}\right)^{-1}, \operatorname{ran} \nabla f=\operatorname{dom} \nabla f^{*}=\operatorname{int} \operatorname{dom} f^{*}$ and $\operatorname{ran} \nabla f^{*}=\operatorname{dom} \nabla f=\operatorname{int} \operatorname{dom} f$.

Recall that a function $f$ is said to be (i) essentially stirctly convex if $(\partial f)^{-1}$ is locally bounded on its domain and $f$ is strictly convex on every convex subset of dom $\partial f$; (ii) essentially smooth if $\partial f$ is both locally bounded and single-valued on its domain; (iii) Legendre, if it is both essentially smooth and essentially strictly convex. Let $f: E \rightarrow(-\infty,+\infty]$ be a Gâteaux differentiable function. The Bregman distance with respect to $f$ is the function $D_{f}: \operatorname{dom} f \times \operatorname{int} \operatorname{dom} f \rightarrow[0,+\infty)$ defined by

$$
D_{f}(y, x):=f(y)-f(x)-\langle\nabla f(x), y-x\rangle .
$$

Recall that bifunction $V_{f}: E \times E^{*} \rightarrow[0, \infty)$ associated with $f$ is defined by

$$
V_{f}\left(x, x^{*}\right)=f^{*}\left(x^{*}\right)+f(x)-\left\langle x, x^{*}\right\rangle, \forall x \in E, x^{*} \in E^{*} .
$$

Then $V_{f}$ is nonnegative and satisfies

$$
V_{f}\left(x, x^{*}\right)=D_{f}\left(x, \nabla f^{*}\left(x^{*}\right)\right), \forall x \in E, x^{*} \in E^{*} .
$$

$D_{f}(\cdot, \cdot)$ has the following important property, called the three point identity. For any $x \in \operatorname{dom} f$ and $y, z \in$ int $\operatorname{dom} f$,

$$
\langle\nabla f(z)-\nabla f(y), x-y\rangle=D_{f}(x, y)-D_{f}(x, z)+D_{f}(y, z) .
$$


Let $f: E \rightarrow(-\infty,+\infty]$ be a convex and Gâteaux differentiable function and let $C \subset \operatorname{dom} f$ be a nonempty, closed, and convex set. The Bregman projection $x \in \operatorname{int} \operatorname{dom} f$ onto $C$ is the unique vector $P_{C}^{f}(x) \in C$ satisfying

$$
D_{f}\left(P_{C}^{f}(x), x\right)=\inf \left\{D_{f}(y, x): y \in C\right\} .
$$

Let $B_{r}:=\{z \in E:\|z\| \leq r\}$ and $S_{E}=\{x \in E:\|x\|=1\}$. Then, a function $f: E \rightarrow \mathbb{R}$ is said to be uniformly convex on bounded subsets of $E$ if $\rho_{r}(t)>0$ for all $r, t>0$, where $\rho_{r}:[0, \infty) \rightarrow[0, \infty]$ is defined by

$$
\rho_{r}(t):=\inf _{x, y \in B_{r},\|x-y\|=t, \alpha \in(0,1)} \frac{\alpha f(x)+(1-\alpha) f(y)-f(\alpha x+(1-\alpha) y)}{\alpha(1-\alpha)} .
$$

Let $f: E \rightarrow(-\infty,+\infty]$ be Gâteaux differentiable. The modulus of total convexity of $f$ at $x \in \operatorname{dom} f$ is the function $v_{f}(x, \cdot):[0,+\infty) \rightarrow[0,+\infty]$ defined by

$$
v_{f}(x, t):=\inf \left\{D_{f}(y, x): y \in \operatorname{domf},\|y-x\|=t\right\} .
$$

The modulus of the total convexity of the function $f$ on the set $B$ is the function $v_{f}$ : int $\operatorname{dom} f \times[0,+\infty) \rightarrow$ $[0,+\infty]$ defined by

$$
v_{f}(B, t):=\inf \left\{v_{f}(x, t): x \in B \cap \operatorname{domf}\right\} .
$$

Recall that a function $f$ is said to be: (i) totally convex at $x$ if $v_{f}(x, t)>0$, whenever $t>0$; (ii) totally convex if it is totally convex at any point $x \in \operatorname{int} \operatorname{dom} f$; (iii) totally convex on bounded sets if $v_{f}(B, t)>0$ for any nonempty bounded subset $B$ of $E$ and $t>0$. A function $f$ is said to be: strongly coercive if $\lim _{\|x\| \rightarrow \infty} f(x) /\|x\|=\infty$; sequentially consistent if for any two sequences $\left\{x_{n}\right\}$ and $\left\{y_{n}\right\}$ in $E$ such that the first one is bounded, $\lim _{n \rightarrow \infty} D_{f}\left(y_{n}, x_{n}\right)=0 \Rightarrow \lim _{n \rightarrow \infty}\left\|y_{n}-x_{n}\right\|=0$. Recall that $T$ is said to be set-valued Bregman quasi-strictly pseudo-contractive with respect to $f$ if $F(T) \neq \emptyset$ and $D_{f}(p, u) \leq D_{f}(p, x)+k D_{f}(x, u), \forall u \in T x, x \in C, p \in F(T)$. Further, $T$ is said to be set-valued Bregman quasi-nonexpansive with respect to $f$ if $F(T) \neq \emptyset$ and $D_{f}(p, u) \leq D_{f}(p, x), \forall u \in T x, x \in C, p \in F(T)$.

Normal Mann iterative method is a powerful scheme to dealing with convex optimization problems; see, e.g., $[10,11,12,13,14]$. One knows that the normal Mann iterative method is efficient for nonexpansive-type mappings, however, it is only weakly convergent in the framework of infinite dimensional spaces. The research on modified Mann iterative method is now under the spotlight of many researchers; see, e.g., $[15,16,17,18,19]$ and the references therein. We have to mention that the success achieved in using geometric properties of Hilbert spaces is not easy to carry out to the framework of Banach spaces. The main difficulty is that the normalized duality map appears in most Banach space inequalities. This creates very serious technical difficulties in computation. Recently, attempts with the Bregman distance have been made to overcome these difficulties; see [19, 20, 21] and the references therein.

We in this paper focus on a Bregan projection algorithm for generalized equilibrium and fixed point problems of a family of closed multi-valued Bregman quasi-strict pseudocontractions. To study equilibrium problem (1.1), we need the following restrictions on bifunction $G$ and operator $M$.

(A1) $G(x, y) \geq \limsup _{t \downarrow 0} G(t z+(1-t) x, y), \forall x, y, z \in C$;

(A2) $G(y, x)+g(x, y) \leq 0, \forall x, y \in C$;

(A3) $G(x, x) \equiv 0, \forall a \in C$;

(A4) $y \mapsto G(x, y)$ is convex and weakly lower semi-continuous, $\forall x \in C$; 
(A5) $M$ is Bregman inverse-strongly monotone (BISM [22]), that is, $\operatorname{dom}(A) \cap \operatorname{int} \operatorname{dom} f \neq \emptyset$ and for any $x, y \in \operatorname{int} \operatorname{dom} f$ and each $x^{\prime} \in M x, y^{\prime} \in M y,\left\langle x^{\prime}-y^{\prime}, \nabla f^{*}\left(\nabla f(x)-x^{\prime}\right)-\nabla f^{*}\left(\nabla f(y)-y^{\prime}\right)\right\rangle \geq 0$.

\section{PRELIMINARIES}

For $r>0$, the resolvent operator of bifunction $G$ and operator $M, \operatorname{Res}_{r}^{M, G}: E \rightarrow C$ is defined as follows:

$$
\operatorname{Res}_{r}^{M, G}(x)=\{z \in C: r\langle M z, y-z\rangle+\langle y-z, \nabla f(z)-\nabla f(x)\rangle+r G(z, y) \geq 0, \forall y \in C\}, \forall x \in E .
$$

From [23], the following lemma is easy to reach.

Lemma 2.1. Let $E$ be a reflexive Banach space. Let $C$ be a nonempty, closed, and convex subset of $E$. Let $f: E \rightarrow \mathbb{R}$ be a convex, continuous, and strongly coercive function which is bounded on bounded subsets and uniformly convex on bounded subsets of $E$ and let $M: C \rightarrow E^{*}$ be a Bregman inverse-strongly monotone operator. Let $M, G$ satisfy $(R-1)-(R-5)$ and let $\operatorname{Res}_{r}^{M, G}: E \rightarrow C$ be the resolvent defined above. Then the following statements hold:

(a) $\operatorname{Res}_{r}^{M, G}$ is single-valued;

(b) $F\left(\operatorname{Res}_{r}^{M, G}\right)=\operatorname{GEP}(M, G)$ is closed and convex;

(c) $D_{f}\left(p, \operatorname{Res}_{r}^{M, G} x\right)+D_{f}\left(\operatorname{Res}_{r}^{M, G} x, x\right) \leq D_{f}(p, x), \forall p \in G E P(M, G), \forall x \in E$.

Lemma 2.2. [24] Suppose $x \in E$ and $y \in$ int domf. If $f$ is essentially strictly convex, then $D_{f}(x, y)=$ $0 \Leftrightarrow x=y$. Function $f$ is sequentially consistent if and only if $f$ is totally convex on bounded sets.

Lemma 2.3. [24] Let $f: E \rightarrow \mathbb{R}$ be a Gâteaux differentiable and totally convex function. If $x_{0} \in E$ and the sequence $\left\{D_{f}\left(x_{n}, x_{0}\right)\right\}$ is bounded, then the sequence $\left\{x_{n}\right\}$ is bounded too.

Lemma 2.4. [24] Let $f: E \rightarrow \mathbb{R}$ be a convex function which is bounded on bounded subsets of $E$. $f^{*}$ is Fréchet differentiable and $\nabla f^{*}$ is uniformly norm-to-norm continuous on bounded subsets of dom $f^{*}=E^{*}$ if and only if $f$ is strongly coercive and uniformly convex on bounded subsets of $E$.

Lemma 2.5. Let $f: E \rightarrow R$ be a Legendre function which is uniformly Fréchet differentiable and bounded on subsets of $E$. Let $C$ be a nonempty, closed, and convex subset of $E$ and let $T: C \rightarrow C B(C)$ be a multivalued Bregman quasi-strictly pseudocontractive mapping with respect to $f$. Then, for any $x \in C, u \in T x$, $p \in F(T)$ and $k \in[0,1),(1-k) D_{f}(x, u) \leq\langle x-p, \nabla f(x)-\nabla f(u)\rangle$.

Proof. Let $u \in T x, p \in F(T), x \in C$, and $k \in[0,1)$, one has

$$
D_{f}(p, u) \leq D_{f}(p, x)+k D_{f}(x, u) .
$$

This implies that

$$
D_{f}(p, x)+D_{f}(x, u)+\langle p-x, \nabla f(x)-\nabla f(u)\rangle \leq D_{f}(p, x)+k D_{f}(x, u) .
$$

It follows that

$$
(1-k) D_{f}(x, u) \leq\langle x-p, \nabla f(x)-\nabla f(u)\rangle .
$$

This completes the proof.

Lemma 2.6. [19] Let $f: E \rightarrow R$ be a Legendre function which is uniformly Fréchet differentiable on bounded subsets of $E$. Let $C$ be a nonempty, closed, and convex subset of $E$ and let $T: C \rightarrow C B(C)$ be a multi-valued Bregman quasi-strictly pseudocontractive mapping with respect to $f$. Then $F(T)$ is a convex and closed set. 
Lemma 2.7. [25] Suppose that $f$ is Gâteaux differentiable and totally convex on int domf. Let $x \in$ int $\operatorname{dom} f$ and let $C \subset$ int domf be a nonempty, closed and convex set. If $\hat{x} \in C$, then the following conditions are equivalent: $(i)\langle\nabla f(x)-\nabla f(\hat{x}), \hat{x}-y\rangle \geq 0, \forall y \in C$; (i) $D_{f}(y, \hat{x})+D_{f}(\hat{x}, x) \leq D_{f}(y, x), \forall y \in C$; and (iii) $\hat{x}=P_{C}^{f}(x)$.

\section{THE STRONG CONVERGENCE THEOREM}

In this section, we state and prove our main theorem.

Theorem 3.1. Let $C$ be a nonempty, convex and closed set in a real reflexive Banach space E. Let $M_{i}$ be a BISM and let Let $G_{i}$ be a bifunction with (A1), (A2), (A3), (A4) and (A5) for each $i \in \Pi$. Let $f: E \rightarrow \mathbb{R}$ be a strongly coercive Legendre function which is bounded, uniformly Fréchet differentiable, and totally convex on bounded subsets of $E$. Let $\prod$ be a index set. Let $T_{i}: C \rightarrow C B(C)$ be a closed and multi-valued Bregman quasi-strict pseudocontraction. Assume that $\Omega:=\cap_{i \in \Pi} F\left(T_{i}\right) \cap \cap_{i \in \Pi} G E P\left(M, G_{i}\right) \neq \emptyset$. Let $\left\{x_{n}\right\}$ be a sequence defined by

$$
\left\{\begin{array}{l}
x_{0} \in E, C_{1, i}=C, C_{1}=\cap_{i \in \Pi} C_{1, i}, x_{1}=P_{C_{1}}^{f}\left(x_{0}\right), \\
y_{n, i}=\nabla f^{*}\left[\alpha_{n, i} \nabla f\left(x_{n}\right)+\left(1-\alpha_{n, i}\right) \nabla f\left(z_{n, i}\right)\right], \quad z_{n, i} \in T_{i} x_{n}, \\
r_{n, i}\left\langle M_{i} u_{n, i}, y-u_{n, i}\right\rangle+r_{n, i} G_{i}\left(u_{n, i}, y\right)+\left\langle y-u_{n, i}, \nabla f\left(u_{n, i}\right)-\nabla f\left(y_{n, i}\right)\right\rangle \geq 0 \\
C_{n+1, i}=\left\{z \in C_{n, i}: D_{f}\left(z, u_{n, i}\right) \leq D_{f}\left(z, y_{n, i}\right) \leq D_{f}\left(z, x_{n}\right)\right. \\
\left.\quad+\frac{\kappa}{1-\kappa}\left\langle x_{n}-z, \nabla f\left(x_{n}\right)-\nabla f\left(z_{n, i}\right)\right\rangle\right\} \\
C_{n+1}=\cap_{i \in \Pi} C_{n+i, i}, \\
x_{n+1}=P_{C_{n+1}}^{f}\left(x_{1}\right),
\end{array}\right.
$$

where $\kappa \in[0,1), \liminf _{n \rightarrow \infty}\left(1-\alpha_{n, i}\right) \alpha_{n, i}>0 \liminf _{n \rightarrow \infty} r_{n, i}>0$, for $\forall i \in \Pi$. Then $\left\{x_{n}\right\}$ converges in norm to $\widehat{p}=P_{\Omega}^{f}\left(x_{1}\right)$, where $P_{\Omega}^{f}$ is the Bregman projection of $E$ onto $\Omega$.

Proof. Using Lemma 2.1 and Lemma 2.6, one concludes that our solution set is convex and closed. Now, we prove that set $C_{n}$ is a convex and closed set. One knows that $C_{1, i}=C$ is a convex and closed set. We now let $C_{k, i}$ is a convex and closed subset for every integer $k \geq 1$. Set $z_{1}$ and $z_{2}$ be two arbitrary points in $C_{m+1, i}$. From the construction of set $C_{n}$, one obtains $z_{1}, z_{2} \in C_{k, i}$. Further, one sets

$$
z_{1,2}=(1-\lambda) z_{2}+\lambda z_{1}
$$

where $\lambda$ is a real number in $(0,1)$. It follows that

$$
\begin{aligned}
D_{f}\left(z_{1,2}, u_{k, i}\right) & \leq D_{f}\left(z_{1,2}, y_{k, i}\right) \\
& \leq D_{f}\left(z_{1,2}, x_{k}\right)+\frac{\kappa}{1-\kappa}\left\langle x_{k}-z_{1,2}, \nabla f\left(x_{k}\right)-\nabla f\left(z_{k, i}\right)\right\rangle .
\end{aligned}
$$

In view of $z_{1,2} \in C_{n, i}$, we obtain that $C_{n, i} \in C_{k+1, i}$. This proves that $C_{k+1, i}$ is a convex and closed set. Hence, $C_{n, i}$ is also a convex and closed set. One concludes that $P_{\Omega}^{f}\left(x_{0}\right)$ is well defined. 
Next, one focuses on $\Omega \subset C_{n}$. Indeed, it is easy to see $\Omega \subset C_{1}=C$. Set $\Omega \subset C_{m, i}$. Note that $u_{m, i}=$ $\operatorname{Res}_{r_{m, i}}^{M_{i}, G_{i}} y_{m}$. For any $w \in \Omega \subset C_{m, i}$, we reach

$$
\begin{aligned}
D_{f}\left(w, u_{m, i}\right)= & f(w)-\left\langle w, \alpha_{m, i} \nabla f\left(x_{m}\right)+\left(1-\alpha_{m, i}\right) \nabla f\left(z_{m, i}\right)\right\rangle \\
& +f^{*}\left(\alpha_{m, i} \nabla f\left(x_{m}\right)+\left(1-\alpha_{m, i}\right) \nabla f\left(z_{m, i}\right)\right) \\
\leq & \alpha_{m} f(w)-\alpha_{m, i}\left\langle w, \nabla f\left(x_{m}\right)\right\rangle+\alpha_{m, i} f^{*}\left(x_{m}\right) \\
& +\left(1-\alpha_{m, i}\right) f(w)-\left(1-\alpha_{m, i}\right)\left\langle w, \nabla f\left(z_{m, i}\right)\right\rangle+\left(1-\alpha_{m}\right) f^{*}\left(\nabla f\left(z_{m, i}\right)\right) \\
\leq & \left(1-\alpha_{m, i}\right)\left[D_{f}\left(w, x_{m}\right)+k D_{f}\left(x_{m}, z_{m, i}\right)\right]+\alpha_{m, i} D_{f}\left(w, x_{m}\right) \\
\leq & \frac{\left(1-\alpha_{m, i}\right) k}{1-k}\left\langle x_{m}-w, \nabla f\left(x_{m}\right)-\nabla f\left(z_{m, i}\right)\right\rangle+D_{f}\left(w, x_{m}\right) \\
\leq & \frac{k\left\langle x_{m}-w, \nabla f\left(x_{m}\right)-\nabla f\left(z_{m, i}\right)\right\rangle}{1-k}+D_{f}\left(w, x_{m}\right),
\end{aligned}
$$

which yields that $w \in C_{m+1, i}$. Hence, $\Omega \subset C_{n, i}$ and then $\Omega \subset C_{n}=\cap_{i \in \Pi} C_{n, i}$. It follows from Lemma 2.7 that

$$
\left\langle y-x_{n}, \nabla f\left(x_{1}\right)-\nabla f\left(x_{n}\right)\right\rangle \leq 0, \quad \forall y \in C_{n},
$$

By virtue of $\Omega \subset C_{n}$, one concludes that

$$
\left\langle w-x_{n}, \nabla f\left(x_{1}\right)-\nabla f\left(x_{n}\right)\right\rangle \leq 0, \quad \forall w \in \Omega .
$$

By using Lemma 2.7 , one has

$$
\begin{aligned}
D_{f}\left(x_{n}, x_{1}\right) & =D_{f}\left(P_{C_{n}}^{f}\left(x_{1}\right), x_{1}\right) \\
& \leq D_{f}\left(w, x_{1}\right)-D_{f}\left(w, P_{C_{n}}^{f}\left(x_{1}\right)\right) \\
& \leq D_{f}\left(w, x_{1}\right),
\end{aligned}
$$

for each $w \in \Omega$. This proves the sequence $\left\{D_{f}\left(x_{n}, x_{1}\right)\right\}$ is a bounded sequence. This further proves $\left\{x_{n}\right\}$ is a bounded iterative sequence too. Note that $D_{f}\left(x_{n+1}, x_{1}\right) \geq D_{f}\left(x_{n}, x_{1}\right)$. So, $\left\{D_{f}\left(x_{n}, x_{1}\right)\right\}$ is a nondecreasing sequence. We conclude that $\lim _{n \rightarrow \infty} D_{f}\left(x_{n}, x_{1}\right)$ exists. Without loss of generality, we may suppose that there exists a subsequence $\left\{x_{n_{j}}\right\} \subset\left\{x_{n}\right\}$ such that $x_{n_{j}} \rightarrow \widehat{p}$, which lies in $C_{n}$. On the other hand, one has

$$
D_{f}\left(x_{n_{j}}, x_{1}\right) \leq D_{f}\left(\widehat{p}, x_{1}\right)
$$

and

$$
\begin{aligned}
D_{f}\left(\widehat{p}, x_{1}\right) & =f(\widehat{p})-f\left(x_{1}\right)-\left\langle\nabla f\left(x_{1}\right), \widehat{p}-x_{1}\right\rangle \\
& \leq \liminf _{j \rightarrow \infty}\left\{f\left(x_{n_{j}}\right)-f\left(x_{1}\right)-\left\langle\nabla f\left(x_{1}\right), x_{n_{j}}-x_{1}\right\rangle\right\} \\
& \leq \liminf _{j \rightarrow \infty} D_{f}\left(x_{n_{j}}, x_{1}\right) .
\end{aligned}
$$

Combining (3.3) and (3.4), one arrives at

$$
D_{f}\left(\widehat{p}, x_{1}\right) \geq \limsup _{j \rightarrow \infty} D_{f}\left(x_{n_{j}}, x_{1}\right) \geq \liminf _{j \rightarrow \infty} D_{f}\left(x_{n_{j}}, x_{1}\right) \geq D_{f}\left(\widehat{p}, x_{1}\right) .
$$

This yields that $D_{f}\left(\widehat{p}, x_{1}\right)=\lim _{j \rightarrow \infty} D_{f}\left(x_{n_{j}}, x_{1}\right)$. Employing Lemma 2.7, one obtains that

$$
D_{f}\left(\widehat{p}, x_{n_{j}}\right)+D_{f}\left(x_{n_{j}}, x_{1}\right) \leq D_{f}\left(\widehat{p}, x_{1} .\right.
$$


Hence, $\lim _{j \rightarrow \infty} D_{f}\left(\widehat{p}, x_{n_{j}}\right)=0$. Using Lemma 2.2 that $\lim _{j \rightarrow \infty} x_{n_{j}}=\widehat{p}$. Since $\left\{D_{f}\left(x_{n}, x_{0}\right)\right\}$ is a convergent sequence, one obtains that

$$
\lim _{n \rightarrow \infty} D_{f}\left(x_{n}, x_{1}\right)=D_{f}\left(\widehat{p}, x_{1}\right)
$$

Using Lemma 2.7, one has

$$
D_{f}\left(\widehat{p}, x_{n}\right)+D_{f}\left(x_{n}, x_{1}\right) \leq D_{f}\left(\widehat{p}, x_{1}\right) .
$$

Further, Lemma 2.2 implies that

$$
\lim _{n \rightarrow \infty} x_{n}=\widehat{p}
$$

Note that

$$
\begin{aligned}
D_{f}\left(x_{n+1}, u_{n, i}\right) & \leq D_{f}\left(x_{n+1}, y_{n, i}\right) \\
& \leq D_{f}\left(x_{n+1}, x_{n}\right)+\frac{\kappa}{1-\kappa}\left\langle x_{n}-x_{n+1}, \nabla f\left(x_{n}\right)-\nabla f\left(z_{n, i}\right)\right\rangle .
\end{aligned}
$$

From the situation that $x_{n} \rightarrow \widehat{p}$ as $n \rightarrow \infty$, one asserts that

$$
\lim _{n \rightarrow \infty} D_{f}\left(x_{n+1}, u_{n, i}\right)=\lim _{n \rightarrow \infty} D_{f}\left(x_{n+1}, y_{n, i}\right)=0 .
$$

It follows that

$$
\lim _{n \rightarrow \infty}\left\|x_{n+1}-u_{n, i}\right\|=\lim _{n \rightarrow \infty}\left\|x_{n+1}-y_{n, i}\right\|=0 .
$$

From (3.7) and (3.8), we have

$$
\lim _{n \rightarrow \infty}\left\|x_{n}-u_{n, i}\right\|=\lim _{n \rightarrow \infty}\left\|x_{n}-y_{n, i}\right\|=0 .
$$

It follows that

$$
\lim _{n \rightarrow \infty}\left\|\nabla f\left(y_{n, i}\right)-\nabla f\left(x_{n}\right)\right\|=0
$$

It follows that

$$
\lim _{n \rightarrow \infty}\left\|\nabla f\left(x_{n}\right)-\nabla f\left(z_{n, i}\right)\right\|=\lim _{n \rightarrow \infty} \frac{1}{1-\alpha_{n}}\left\|\nabla f\left(x_{n}\right)-\nabla f\left(y_{n, i}\right)\right\|=0 .
$$

Using Lemma 2.5 yields that $\lim _{n \rightarrow \infty}\left\|z_{n, i}-x_{n}\right\|=0$. Therefore $\lim _{n \rightarrow \infty} z_{n, i}=\lim _{n \rightarrow \infty} x_{n}=\widehat{p}$. From the closedness of each $T_{i}$ and $z_{n, i} \in T_{i} x_{n}$, one sees $\widehat{p} \in F\left(T_{i}\right)$. Hence, $\widehat{p} \in \cap_{i \in \Pi} F\left(T_{i}\right)$.

Next, we prove $\widehat{p} \in \cap_{i \in \Pi} G E P\left(M_{i}, G_{i}\right)$. Note that

$$
\left\|y_{n, i}-u_{n, i}\right\| \leq\left\|x_{n}-u_{n, i}\right\|+\left\|x_{n}-y_{n, i}\right\| .
$$

By using (3.9), we have $\lim _{n \rightarrow \infty}\left\|y_{n, i}-u_{n, i}\right\|=0$. Since $\nabla f$ is uniformly norm-to-norm continuous on bounded subsets of $E$, one has

$$
\lim _{n \rightarrow \infty} \frac{\left\|\nabla f\left(y_{n, i}\right)-\nabla f\left(u_{n, i}\right)\right\|}{r_{n, i}}=0 .
$$

From the definition of the resolvent of the generalized equilibrium problem, we have $u_{n, i}=\operatorname{Res}_{r_{n, i}}^{M_{i}, G_{i}} y_{n, i}$. Hence,

$$
r_{n, i}\left\langle M u_{n, i}, y-u_{n, i}\right\rangle+r_{n, i} g_{i}\left(u_{n, i}, y\right)+\left\langle y-u_{n, i}, \nabla f\left(u_{n, i}\right)-\nabla f\left(y_{n, i}\right)\right\rangle \geq 0, \quad \forall y \in C .
$$

Hence, one has

$$
\begin{aligned}
\left\|y-u_{n, i}\right\| \frac{\left\|\nabla f\left(y_{n, i}\right)-\nabla f\left(u_{n, i}\right)\right\|}{r_{n, i}} & \geq \frac{\left\langle y-u_{n, i}, \nabla f\left(u_{n, i}\right)-\nabla f\left(y_{n, i}\right)\right\rangle}{r_{n, i}} \\
& \geq W_{i}\left(y, u_{n, i}\right), \quad \forall y \in C,
\end{aligned}
$$

where

$$
W_{i}\left(y, u_{n, i}\right)=\left\langle M u_{n, i}, y-u_{n, i}\right\rangle+G\left(u_{n, i}, y\right) .
$$


It follows from (3.12) that $W_{i}(y, \widehat{p}) \leq 0, \forall y \in C$. Let

$$
y_{t_{i}}=t_{i} y+\left(1-t_{i}\right) \widehat{p}
$$

where $y \in C$, and $t_{i} \in(0,1)$. It follows that $W_{i}\left(y_{t_{i}}, \widehat{p}\right) \leq 0$. Hence

$$
t_{i} W_{i}\left(y_{t_{i}}, y\right) \geq t_{i} W_{i}\left(y_{t_{i}}, y\right)+\left(1-t_{i}\right) W_{i}\left(y_{t_{i}}, p\right) \geq W_{i}\left(y_{t_{i}}, y_{t_{i}}\right)=0 .
$$

This implies $W_{i}\left(y_{t}, y\right) \geq 0, \forall y \in C$ and then $W_{i}(\widehat{p}, y) \geq 0, \forall y \in C$. Hence

$$
\widehat{p} \in \cap_{i \in \Pi} E P\left(W_{i}\right)=\operatorname{GEP}\left(M_{i}, G_{i}\right) .
$$

This proves that $\widehat{p} \in \Omega$. Finally, we take $n \rightarrow \infty$ in (3.2) to obtain that

$$
\left\langle w-\widehat{p}, \nabla f\left(x_{1}\right)-\nabla f\left(x_{n}\right)\right\rangle \leq 0, \quad \forall w \in \Omega .
$$

Using Lemma 2.7, one has $\widehat{p}=P_{\Omega}^{f}\left(x_{1}\right)$. This completes the proof.

If $f(x)=\|x\|^{2}, \forall x \in E$, then the class of multi-valued Bregman quasi-strict pseudo-contractions is reduced to the class of multi-valued quasi-strict pseudo-contractions [26]. We have the following result.

Corollary 3.2. Let $C$ be a nonempty, convex and closed set in a real reflexive Banach space E. Let $M_{i}$ be a BISM and let Let $G_{i}$ be a bifunction with (A1), (A2), (A3), (A4) and (A5) for each $i \in \prod$. Let $f: E \rightarrow \mathbb{R}$ be a strongly coercive Legendre function which is bounded, uniformly Fréchet differentiable, and totally convex on bounded subsets of $E$. Let $\prod$ be a index set. Let $T_{i}: C \rightarrow C B(C)$ be a closed and multi-valued Bregman quasi-strict pseudocontraction. Assume that $\Omega:=\cap_{i \in \Pi} F\left(T_{i}\right) \cap \cap_{i \in \Pi} G E P\left(M, G_{i}\right) \neq \emptyset$. Let $\left\{x_{n}\right\}$ be a sequence defined by

$$
\left\{\begin{array}{l}
x_{0} \in E, C_{1, i}=C, C_{1}=\cap_{i \in \Pi} C_{1, i}, x_{1}=P_{C_{1}}^{f}\left(x_{0}\right), \\
y_{n, i}=J^{-1}\left[\alpha_{n, i} J\left(x_{n}\right)+\left(1-\alpha_{n, i}\right) J\left(z_{n, i}\right)\right], \quad z_{n, i} \in T_{i} x_{n}, \\
r_{n, i}\left\langle M_{i} u_{n, i}, y-u_{n, i}\right\rangle+r_{n, i} G_{i}\left(u_{n, i}, y\right)+\left\langle y-u_{n, i}, J\left(u_{n, i}\right)-J\left(y_{n, i}\right)\right\rangle \geq 0, \\
C_{n+1, i}=\left\{z \in C_{n, i}: D_{f}\left(z, u_{n, i}\right) \leq D_{f}\left(z, y_{n, i}\right) \leq D_{f}\left(z, x_{n}\right)\right. \\
\left.\quad \quad+\frac{\kappa}{1-\kappa}\left\langle x_{n}-z, J\left(x_{n}\right)-J\left(z_{n, i}\right)\right\rangle\right\}, \\
C_{n+1}=\cap_{i \in \Pi} C_{n+i, i}, \\
x_{n+1}=P_{C_{n+1}}^{f}\left(x_{1}\right),
\end{array}\right.
$$

where $\kappa \in[0,1), \liminf _{n \rightarrow \infty}\left(1-\alpha_{n, i}\right) \alpha_{n, i}>0 \liminf _{n \rightarrow \infty} r_{n, i}>0$, for $\forall i \in \Pi$. Then $\left\{x_{n}\right\}$ converges strongly to $\widehat{p}=P_{\Omega}^{f}\left(x_{1}\right)$, where $P_{\Omega}^{f}$ is the generalized projection of $E$ onto $\Omega$.

\section{Funding}

This paper was supported by the National Natural Science Foundation of China under Grant 11601348.

\section{Acknowledgements}

The author is grateful to the referees for useful suggestions which improved the contents of this paper. 


\section{REFERENCES}

[1] S. Takahashi, W. Takahashi, Strong convergence theorem for a generalized equilibrium problem and a nonexpansive mapping in a Hilbert space, Nonlinear Anal. 69 (2008), 1025-1033.

[2] E. Blum, W. Oettli, From optimization and variational inequalities to equilibrium problems, Math. Stud. 63 (1994), 123-145.

[3] C.E. Chidume, O.M. Romanus, U.V. Nnyaba, An iterative algorithm for solving split equilibrium problems and split equality variational inclusions for a class of nonexpansive-type maps, Optimization, 67 (2018), 1949-1962.

[4] U.V. Nnyaba, O.M. Romanus, New algorithms for solving system of generalized mixed equilibrium problems and some nonlinear problems, with applications, Optimization, 67 (2018), 2325-2345.

[5] X. Qin, Y.J. Cho, S.M. Kang, Convergence theorems of common elements for equilibrium problems and fixed point problems in Banach spaces, J. Comput. Appl. Math. 225 (2009), 20-30.

[6] X. Qin, S.S. Chang, Y.J. Cho, Iterative methods for generalized equilibrium problems and fixed point problems with applications, Nonlinear Anal. 11 (2010), 2963-2972.

[7] V. Dadashi, O.I. Lyiola, Y. Shehu, The subgradient extragradient method for pseudomonotone equilibrium problems, Optimization, doi: 10.1080/02331934.2019.162589.

[8] N.T.T. Thuy, P.T. Hieu, A hybrid method for solving variational inequalities over the common fixed point sets of infinite families of nonexpansive mappings in Banach spaces, Optimization, doi: 10.1080/02331934.2019.1647201.

[9] B.A. Bin Dehaish, X. Qin, A. Latif, H.O. Bakodah, Weak and strong convergence of algorithms for the sum of two accretive operators with applications, J. Nonlinear Convex Anal. 16 (2015), 1321-1336.

[10] K.R. Kazmi, R. Ali, M. Furkan, Krasnoselski-Mann type iterative method for hierarchical fixed point problem and split mixed equilibrium problem, Numer. Algo. 77 (2018), 289-308.

[11] S.Y. Cho, B.A. Bin Dehaish, X. Qin, Weak convergence of a splitting algorithm in Hilbert spaces, J. Appll. Anal. Comput. 7 (2017), 427-438.

[12] S.Y. Cho, Generalized mixed equilibrium and fixed point problems in a Banach space, J. Nonlinear Sci. Appl. 9 (2016), 1083-1092.

[13] X. Qin, A. Petrusel, J.C. Yao, CQ iterative algorithms for fixed points of nonexpansive mappings and split feasibility problems in Hilbert spaces, J. Nonlinear Convex Anal. 19 (2018), 157-165.

[14] X. Qin, J.C. Yao, Weak convergence of a Mann-like algorithm for nonexpansive and accretive operators, J. Inequal. Appl. 2016 (2016), Article ID 232.

[15] Q.L. Dong, Y.J. Cho, T.M. Rassias, General Inertial Mann Algorithms and Their Convergence Analysis for Nonexpansive Mappings. In: Rassias T. (eds) Applications of Nonlinear Analysis. Springer Optimization and Its Applications, vol 134. Springer, Cham, 2018.

[16] S. Vong, D. Liu, An inertial Mann algorithm for nonexpansive mappings, J. Fixed Point Theory Appl. 20 (2018), 102.

[17] X. Qin, J.C. Yao, A viscosity iterative method for a split feasibility problem, J. Nonlinear Convex Anal. 20 (2019), 14971506.

[18] G. Marino, et al., Strong convergence of the modified Mann iterative method for strict pseudo-contractions, Comput. Math. Appl. 57 (2009), 455-465.

[19] Z.M. Wang, A. Wei, Some results on a finite family of Bregman quasi-strict pseudo-contractions, J. Nonlinear Sci. Appl. 10 (2017), 975-989.

[20] Z.M. Wang, A. Wei, P. Kumam, Hybrid Bregman projection methods for fixed point and equilibrium problems, Carpathian J. Math. 34 (2018), 441-447.

[21] Z. Wang, Strong convergence theorems for Bregman quasi-strict pseudo-contractions in reflexive Banach spaces with applications, Fixed Point Theory Appl. 2015 (2015), 91.

[22] S. Reich, S. Sasbach, Two strong convergence theorems for Bregman strongly nonexpansive operators in reflexive Banach spaces, Nonlinear Anal. 73 (2010), 122-135.

[23] S.S. Chang, H.W.J. Lee, C.K. Chan, A new hybrid method for solving a generalized equilibrium problem, solving a variational inequality problem and obtaining common fixed points in Banach spaces, with applications, Nonlinear Anal. 73 (2010), 2260-2270. 
[24] H.H. Bauschke, J.M. Borwein, P.L. Combettes, Essential smoothness, essential strict convexity, and Legendre functions in Banach spaces, Commun. Contemp. Math. 3 (2001), 615-664.

[25] D. Butnariu, E. Resmerita, Bregman distances, totally convex functions and a method for solving operator equations in Banach spaces, Abstr. Appl. Anal. 2006 (2006), Art. ID 84919.

[26] H. Zhou, X. Gao, An iterative method of fixed points for closed and quasi-strict pseudo-contractions in Banach spaces, J. Appl. Math. Comput. 33 (2010), 227-237. 